\title{
Pour un modèle de l'acquisition des liaisons basé sur l'usage: trois études de cas
}

\author{
JEAN-PIERRE CHEVROT \\ Université Grenoble 3 \\ DAMIEN CHABANAL \\ Université Blaise Pascal, Clermont-Ferrand \\ CÉLINE DUGUA \\ Université Grenoble 3
}

(Received August 2005; revised August 2006)

RESUME

Des études récentes, essentiellement fondées sur des expérimentations, ont précisé les relations entre l'usage enfantin des liaisons, les erreurs commises par les enfants et la segmentation des mots nouveaux. Alors que les auteurs s'accordent sur les faits, ils divergent sur les facteurs explicatifs susceptibles de rendre compte des étapes développementales. Chevrot, Dugua et Fayol (2005) défendent une approche basée sur l'usage alors que Wauquier-Gravelines (2005) et Wauquier-Gravelines et Braud (2005) soutiennent une conception dans laquelle le développement est guidé par les principes universels de la grammaire. Les données issues de trois études de cas confirment et clarifient les résultats expérimentaux. Deux types d'effets de fréquence soutiennent l'approche basée sur l'usage.

\section{INTRODUCTION ${ }^{1}$}

La liaison est considérée depuis 40 ans comme un des phénomènes vedettes de la phonologie du français (Tranel, 2000). Elle a servi de test à la majorité des modèles génératifs et son fonctionnement a été décrit à travers plusieurs études de corpus.

Les erreurs de liaison commises par l'enfant sont remarquées depuis longtemps (Grégoire, I937). Elles consistent généralement à ajouter une liaison ([lənurs] pour l'ours), à produire une liaison erronée ([dønurs] pour deux ours) ou à omettre une liaison ([døurs] pour deux ours). Ces erreurs ont parfois été mises à contribution pour éclairer le traitement phonologique adulte (Klausenburger,

${ }^{1}$ Les travaux présentés ont été soutenus par l'ACI Cognitique et par le programme Emergence de la Région Rhône-Alpes. Nous remercions Marie-Hélène Côté, Michel Fayol, Bernard Laks, Yves Charles Morin et Sophie Wauquier-Gravelines pour l'impulsion qu'ils ont donnée à notre réflexion. Nous remercions aussi Sophie, Esther et tous les autres enfants. Sans leurs erreurs de liaison, cette recherche n'aurait pas débuté. 
I974; Gaatone, I979). En revanche, il a fallu attendre ces dernières années pour que le processus d'acquisition des liaisons fasse l'objet d'études empiriques et de propositions théoriques cohérentes (Chevrot et Fayol, 200 ; Dugua, 2002; Morin, 2003 [I998]; Chabanal, 2003; Nardy, 2003; Chevrot, Dugua et Fayol, 2005; Côté, 2005; Wauquier-Gravelines, 2005; Wauquier-Gravelines et Braud, 2005).

Ces travaux s'accordent pour l'essentiel sur les faits qui jalonnent l'évolution des productions enfantines. Toutefois, ils opposent deux conceptions théoriques quand il s'agit d'avancer des principes explicatifs des forces qui sous-tendent le développement. D'un côté, Chevrot, Dugua et Fayol (2005) adoptent un point de vue exemplariste et basé sur l'usage (Barlow et Kemmer, 2000; Bybee, 200 I, 2005; Tomasello, 2003), selon lequel les généralisations formulées par l'enfant émergent de l'accumulation, du traitement analogique et de l'organisation d'un matériel linguistique concret (mots et séquences de mots) mémorisé précocement. A l'opposé, Wauquier-Gravelines (2005) et Wauquier-Gravelines et Braud (2005) considèrent que l'enfant construit une représentation phonologique abstraite de la liaison en exploitant, dans le cadre d'une grammaire en voie de paramétrisation, sa connaissance des contraintes phonologiques universelles et des régularités morphologiques.

Nous commencerons par rappeler brièvement le fonctionnement de la liaison chez l'adulte et les difficultés qu'elle occasionne lors du processus précoce de segmentation et de formation des mots. Nous exposerons ensuite comment chacune des deux conceptions théoriques décrit et explique la trajectoire développementale de l'enfant confronté à ces difficultés. Nous cernerons les traits qui réunissent ces deux conceptions et ceux qui les opposent et nous dégagerons des prédictions empiriques susceptibles de les départager.

La plupart des résultats présentés dans les travaux antérieurs sont fondés sur des tâches expérimentales induisant la production de liaisons à l'intérieur du syntagme nominal. Notre contribution empirique exploitera au contraire des données recueillies en situation naturelle d'interaction: l'étude transversale de cinq enfants âgés de 3 à I I ans, le suivi longitudinal, entre 40 et 50 mois, de deux enfants issus de milieux sociaux contrastés et l'étude d'un corpus d'erreurs recueilli chez une fillette entre 2 et 6 ans. Le recours à des situations d'observation diversifiées ouvre l'éventail des contextes de liaison recueillis et élimine l'éventualité de biais expérimentaux. A la lumière de cette extension des données, nous préciserons les étapes développementales et nous discuterons l'opposition théorique entre une approche basée sur l'usage et une approche fondée sur la connaissance de principes généraux structurant les langues du monde.

\section{LA LIAISON ET LA FORMATION PRECOCE DES MOTS}

Chez l'adulte, la liaison se manifeste dans la parole par l'apparition d'une consonne entre deux mots (dorénavant: moti et mot2). Une condition nécessaire à son apparition est que le mot 2 commence par une voyelle lorsqu'il est prononcé isolément. En revanche, cette consonne n'est jamais présente à la finale du mot I 


\section{L'acquisition des liaisons}

lorsqu'il est situé à la fin d'un énoncé, ni à l'initiale du mot2 lorsqu'il occupe la première position d'un énoncé. Lorsque cette consonne de liaison est produite, elle forme généralement une syllabe avec la voyelle qui suit ${ }^{2}$. Par exemple, un /z/ est prononcé entre les mots les et écureuil dans la séquence les écureuils, avec une syllabification [le.ze.ky.rœj]. Mais, chez l'adulte, ce /z/ n'est prononcé ni dans les veaux ([levo]), ni dans Regarde les! ([rgardle]), ni à l'initiale de l'apostrophe Ecureuil!

Toutes les consonnes ne peuvent pas jouer le rôle de liaison. Une étude de Boë et Tubach (I992) sur 20 heures de parole adulte montre que /n/, /z/ et /t/ représentent $99,7 \%$ des liaisons réalisées, les occurrences restantes étant réparties entre $/ \mathrm{p} /, / \mathrm{r} /$ et $/ \mathrm{d} /$. La possibilité de produire une liaison ainsi que sa nature phonétique dépendent du moti. Par exemple, les motsi un ou aucun déclenchent une liaison en $/ \mathrm{n} /$, les mots I petit ou grand une liaison en /t/, les mots I gros ou deux une liaison en $/ \mathrm{z} /$, alors que les mots I joli ou beau au singulier n'en déclenchent aucune.

Enfin, les auteurs classent les contextes de liaison en trois catégories, définies à partir de critères morphosyntaxiques ou lexicaux: les contextes où la liaison est obligatoire, ceux où elle est variable et ceux où elle est interdite. Plusieurs études de corpus (Ågren, I973; Malécot, I975; Lucci, I983; de Jong; I994; Moisset, 2000) trouvent que la fréquence des liaisons variables est sensible à des facteurs de nature linguistique (longueur, catégorie et fréquence lexicale du motı, débit) et à des facteurs sociolinguistiques (statut social du locuteur, style, âge, sexe), même si les résultats ne sont pas toujours stables d'une étude à l'autre.

Cette présentation de la liaison fait apparaître une double particularité compliquant singulièrement la tâche du jeune enfant qui, pour construire un lexique, doit récupérer la forme, le sens et la fonction communicative des mots dans le flux de la parole environnante.

Premièrement, du fait de l'enchaînement, la consonne de liaison forme une syllabe de type Consonne-Voyelle (CV) avec l'initiale du mot suivant (les arbres est syllabé [le.zarbr]) et cette resyllabisation aboutit à disjoindre la frontière lexicale (située après le/z/) et la frontière syllabique (située avant le/z/). On sait par ailleurs que les processus de reconnaissance lexicale exploitent la correspondance probable entre frontière de syllabes et frontière de mots, aussi bien chez l'adulte (Content, Kearns et Frauenfelder, 200I) que chez les bébés (Mattys et Jusczyk, 200I). Si l'enfant maintient cette stratégie de segmentation dans les contextes liés contenant des mots nouveaux, il placera une frontière lexicale avant la consonne de liaison, qui, de ce fait, sera rattachée à l'initiale de la représentation lexicale du mot qui suit dans la chaîne parlée. Il est donc attendu que l'enfant récupère la forme lexicale/zarbr/à partir de l'audition d'une séquence comme les arbres.

2 Il existe des liaisons réalisées et non enchaînées, surtout dans le discours public, notamment politique (Lucci, I983; Encrevé, 1998). Ces liaisons jouent sans doute un rôle dans l'apprentissage des registres formels. Parce qu'elles rendent manifeste la frontière entre deux mots, elles sont susceptibles de modifier tardivement les représentations lexicales. Nous ferons l'hypothèse qu'elles n'influencent pas le processus développemental précoce, du fait de leur rareté dans l'environnement langagier des jeunes enfants. 
Deuxièmement, la nature phonétique de la consonne de liaison étant déterminée par le moti «comme si elle lui appartenait »(Tranel, 2000), l'enfant rencontre nécessairement chaque mot I suivi d'une consonne de liaison spécifique. Ainsi, dans l'environnement langagier enfantin, un sera régulièrement suivi de la liaison $/ \mathrm{n} /$, des sera suivi de /z/, etc. Cette seconde caractéristique inclinera l'enfant à établir un lien entre la nature phonétique de la liaison et le mot précédent.

Deux contraintes précoces et contradictoires tirent donc l'attachement lexical de la consonne de liaison dans des directions opposées: les contingences distributionnelles entre le mot précédent et la nature phonétique de la liaison l'attirent vers la gauche tandis que la syllabisation de la chaîne parlée la pousse vers la droite. L'issue et la forme du développement dépendront de la résolution de ce conflit, auquel peuvent également participer deux autres forces: d'une part la morphologie, puisqu'il existe une relation entre certaines liaisons et les dérivés ou les formes fléchies du moti (par exemple, entre la liaison /t/ de petit et petitesse, petite); d'autre part, plus tardivement, le contact avec la forme orthographique, puisque la graphie des mots I finit très généralement par une lettre correspondant à la liaison.

A partir de ces ingrédients, deux scénarios développementaux ont été proposés pour rendre compte de la relation entre acquisition des liaisons et formation des mots. Ils s'opposent sur la façon dont ils motivent et ordonnent l'intervention des trois premières des quatre forces en présence mentionnées ci-dessus. L'impact de la graphie sur la liaison (Laks, 2005) ne sera pas observé directement dans cet article.

\section{DEUX CONCEPTIONS THEORIQUES}

\subsection{Une conception exemplariste et basée sur l'usage}

Le premier scénario développemental a été initié par Morin (2003 [I998]) dans le cadre d'une réflexion sur la liaison prénominale. Il a été explicité et documenté ultérieurement par Chevrot, Dugua et Fayol (2005), qui résument les résultats de nombreuses expérimentations réalisées auprès d'enfants de 2 à 6 ans (Chevrot et Fayol, 200I; Dugua, 2002, 2005; Chabanal, 2003; Nardy, 2003). Il semblerait que les grandes lignes de ce scénario puissent être étendues à d'autres types de liaisons obligatoires, notamment celles qui suivent un pronom clitique.

Lors d'une première étape, la disjonction entre frontière lexicale et frontière syllabique induite par la liaison conduirait le jeune enfant à segmenter et mémoriser précocement plusieurs variantes lexicales de chaque mot2. En effet, l'enfant rencontre chaque mot 2 précédé de différentes consonnes de liaison, par exemple, arbre précédé de /n/ dans un arbre, de /z/ dans les arbres, de /t/ dans petit arbre. S'il maintient une segmentation syllabique dans ces différentes occurrences (õe.narbr, le.zarbr, pti.tarbr), il finira par mémoriser plusieurs exemplaires de chaque unité lexicale (/narbr/, /zarbr/, /tarbr/ dans le cas du mot arbre). A ces exemplaires à

initiale consonantique s'ajouterait dès 2 ans (Dugua, 2002), la variante à initiale 
vocalique (/arbr/) de certains mots2 entendus en isolation ou dans des contextes où ils sont précédés d'un moti n'induisant aucune liaison (joli arbre) ou induisant une liaison variable non réalisée (gros arbre entendu sans la liaison /z/).

Ce jeu de variantes lexicales étant établi, la seconde étape du processus d'acquisition consisterait à mémoriser les relations entre certains mots I et la variante adéquate du mot2. L'enfant apprendrait que l'exemplaire /narbr/ suit le mot un, que /zarbr/ suit le mot des, etc. La cause de cet apprentissage serait l'exposition aux séquences moti-mot2 bien formées rencontrées dans l'environnement langagier. C'est donc l'usage du langage en réception qui contribuerait principalement à structurer ce réseau d'associations entre des mots I particuliers et des variantes des mots2.

Une troisième étape serait caractérisée par l'émergence d'une structure plus abstraite résultant de la réanalyse des représentations de l'étape précédente. Pour Morin (2003 [1998], 2005), cette structure prendrait, dans le cas des liaisons prénominales, la forme d'un système productif de préfixes du nom, dont la fonction grammaticale serait de noter une combinaison de nombre et d'état construit. Alternativement, on peut considérer que l'état final du développement est l'élaboration de constructions complexes intégrant la liaison, des items lexicaux et des catégories morphologiques et grammaticales (Bybee, 200I, 2005). Dans le cadre de cette seconde option, l'enfant généraliserait le lien entre un moti particulier et une classe d'exemplaires de mots2 en formant des constructions basées sur des mots particuliers. En mettant en relation les séquences des + /zurs/, des +/zan/, des + /zami/ typiques de l'étape précédente, il élaborerait un schéma de type des $+/ \mathrm{zX} /$, qui lie le déterminant indéfini pluriel à la classe des variantes commençant par /z/. Ce schéma lui permettrait de produire des liaisons correctes sans devoir rencontrer et mémoriser l'ensemble des combinaisons possibles entre les déterminants du français et les variantes des mots2. Plus généralement, ces schémas seraient un cas particulier de constructions basées sur des items, qui sont caractéristiques du développement syntaxique entre 18 et 36 mois et peuvent évoluer vers davantage d'abstraction (Tomasello, 2000, 2003). Ainsi ces schémas préfigureraient la structure interne du groupe nominal.

$\mathrm{Si}$ on admet que les constructions porteuses de liaisons sont en compétition avec des schémas plus généraux de type des $+/ \mathrm{X} /$ ou les $+/ \mathrm{X} /$ qui n'incluent aucune information sur la liaison, ce modèle développemental rend compte de l'ensemble des erreurs produites par les enfants. Ainsi, l'insertion de l'exemplaire / narbr/ dans le schéma des $+/ \mathrm{X} /$ aboutit à la classique erreur de substitution [denarbr] (des arbres avec /n/ au lieu du /z/ attendu). L'insertion de l'exemplaire /arbr/ à voyelle initiale dans le schéma des $+/ \mathrm{X} /$ produit une erreur d'omission de liaison [dearbr] (des arbres prononcé sans liaison). Enfin, les erreurs de remplacement d'un /n/ ou d'un /z/ à l'initiale par une consonne compatible avec le motI - des nombrils prononcé [dezõbril] avec /z/ au lieu de /n/ - résultent de l'insertion de nombril au schéma des $+/ z X /$. Il a été montré que ce dernier type d'erreurs présentait un profil développemental typique des surgénéralisations: leur fréquence augmente vers $4-5$ ans puis diminue vers $5-6$ ans (Chevrot, Dugua et Fayol, 2005). Ce résultat 
conforte l'existence de schémas porteurs de liaison et atteste de leur productivité. Grâce à ces schémas, un enfant qui n'aurait pas mémorisé l'exemplaire /zarbr/ à défaut d'avoir rencontré assez souvent le mot arbre précédé de la liaison $/ \mathrm{z} /$, pourra produire correctement la séquence des arbres. A partir des exemplaires dont il dispose - /narbr/, /zami/, /zan/, etc. - il créerait un exemplaire /zarbr/ afin de satisfaire au schéma des $+/ z \mathrm{X} /$. Lorsqu'il applique à tort ce processus de création analogique à la séquence des nombrils, il aboutit à l'erreur [dezõbril].

\subsection{Une conception basée sur la connaissance de principes universaux}

Wauquier-Gravelines et Braud (2005) s'opposent à cette conception exemplariste et basée sur l'usage. Elles soutiennent que les généralisations de l'enfant ne procèdent pas au coup par coup, avec l'accumulation du matériel lexical, mais sont guidées par les principes universels de la grammaire et par la morphologie. Le canevas développemental qu'elles proposent se déroule en quatre stades. Selon leur conception, l'acquisition des liaisons obligatoires et celle des déterminants sont intrinsèquement liées. En revanche, l'apprentissage des liaisons facultatives est conçu différemment, comme un processus purement lexical.

Le stade le plus précoce est antérieur à la première étape de Chevrot, Dugua et Fayol (2005). Il s'agit d'un état où déterminant et nom ne sont pas encore segmentés, mais intégrés dans un gabarit lexical (Vihman, 1996) qui joue le rôle d'unité minimale. C'est dans la position gauche de ce gabarit que se trouverait le germe de ce qui deviendra plus tard un déterminant autonome. Dans les productions enfantines, ce germe peut se réduire à une voyelle ([รejã] pour un éléphant) mais peut aussi correspondre à l'intégralité du matériel phonétique du déterminant (séquence non segmentée [õnarbr] pour un arbre). Il n'existe pas d'erreurs de liaison à ce stade.

Lors du second stade, l'enfant détermine la frontière entre le déterminant et le nom. Cette segmentation opère conformément au Principe de Maximisation des Attaques, qui rend compte du fait que toute suite VCV est syllabée prioritairement V.CV. Pour privilégier l'occurrence de syllabes CV «non marquées » à l'initiale du nom, l'enfant rattache la position consonantique occupée par la liaison à l'attaque du mot2. Toutefois, la nature phonétique de cette position consonantique initiale n'est pas encore fixée car «à ce stade, l'enfant sait qu'il faut une consonne mais ne sait pas laquelle » (Wauquier-Gravelines et Braud, 2005: 62). Il utilise donc différents moyens pour assigner un contenu concret à cette position d'attaque abstraite: harmonie avec une consonne interne au nom (un éléphant produit [lelefã]), utilisation d'une semi-voyelle par défaut, utilisation probabiliste des liaisons entendues dans cette position. Dans ce cadre, et contrairement aux propositions de Chevrot, Dugua et Fayol (2005), les substitutions de liaison, par exemple un arbre prononcé [õzarbr], ne proviennent pas de l'insertion d'une des variantes du mot2 à un schéma de type un $+/ \mathrm{X} /$. Au contraire, l'erreur [õzarbr] survient parce que l'enfant assigne le contenu phonétique $[\mathrm{z}]$ à la position consonantique abstraite qui est encodée à l'initiale de la représentation phonologique unique de arbre. 
Le troisième stade voit disparaitre les erreurs sur les liaisons obligatoires sous l'impact de la morphologie. L'enfant encoderait les liaisons sous forme de segments flottants à la finale des déterminants et des adjectifs quand il découvre «que le déterminant un porte une consonne nasale au féminin, que les adjectifs comme petit, grand, gros portent une consonne lors d'opérations d'inflexion (petite, grande, grosse) ou de dérivation nominale (petitesse, grandeur, grandir, grosseur, grossir) » (WauquierGravelines et Braud, 2005: 62).

Lors de la quatrième étape, l'enfant continuerait à acquérir les liaisons facultatives pour lesquelles les deux auteurs concèdent un mode d'apprentissage procédant mot par mot.

\subsection{Divergences entre les modèles et prédictions}

Les deux conceptions exposées ci-dessus divergent sur plusieurs points, dont certains permettent des prédictions empiriques susceptibles de les départager. Une divergence mineure concerne l'existence d'un stade très précoce, défendu par Wauquier-Gravelines et Braud (2005), pendant lequel les séquences déterminant + nom fonctionneraient comme des unités non segmentées de stockage et de production. Ce stade serait antérieur à la première étape du scénario de Chevrot, Dugua et Fayol (2005) et n'entre pas en contradiction avec leurs propositions. De fait, dans les théories basées sur l'usage, il est admis que l'enfant mémorise précocement des séquences globales dans lesquelles il aménage progressivement des emplacements libres où peuvent s'insérer des éléments nouveaux (Lieven, Behrens, Speares et Tomasello, 2003). On peut penser que le lexique précoce inclut de telles séquences, coexistant avec des nominaux directement segmentés du fait de leur saillance perceptive ou référentielle ou du fait des distributions dans lesquelles l'enfant les rencontre (noms entendus en apostrophe, par exemple).

Une première opposition irréductible entre les deux modèles concerne la forme des représentations lexicales caractéristiques de l'étape suivante. Dans le modèle exemplariste de Chevrot, Dugua et Fayol (2005), la représentation lexicale multiple associée à l'entrée lexicale du nom ours est la combinaison de plusieurs séquences concrètes - /urs/, /nurs/, /zurs/, /turs/ - sans doute reliées au même contenu sémantique et pragmatique. Le matériel segmental correspondant aux consonnes de liaison appartient donc en propre à ces traces lexicales du nom. Pour Wauquier-Gravelines et Braud, l'enfant encode une position $\mathrm{C}$ abstraite au début de la représentation lexicale unique du mot ours. Cette position abstraite est une composante du nom, mais elle est démunie de contenu phonétique. Lors de la production, l'enfant assigne un contenu phonétique à cette position abstraite par des moyens contextuels, statistiques ou par défaut. Mais en aucun cas ce contenu phonétique n'est lié au nom qui suit et ne lui appartient en propre. Ainsi, toute tendance empirique indiquant une relation entre la nature phonétique des liaisons enfantines $-/ \mathrm{n} /, / \mathrm{z} /$ ou $/ \mathrm{t} /$ - et le nom qui suit milite en faveur des conceptions exemplaristes et basées sur l'usage. C'est notre première prédiction. 
La seconde différence irréductible opposant les deux modèles touche à l'apprentissage premier des liaisons correctes. Pour Chevrot, Dugua et Fayol (2005), les enfants commencent par mémoriser les relations standard entre certains mots I et l'exemplaire adéquat du mot2: $u n+/$ nurs/, deux $+/$ zurs/, etc. De ce fait, ils devraient réaliser davantage de liaisons correctes dans les séquences moti-mot2 qu'ils entendent et utilisent souvent. Pour Wauquier-Gravelines et Braud (2005), l'enfant encode une consonne flottante à la finale du mot I sous l'impact des relations morphologiques entre ce mot et ses dérivés ou ses formes fléchies. Ce processus dépend de l'existence de dérivés ou de formes fléchies du mot I et de leur fréquence, mais en aucun cas il ne dépend de la probabilité des séquences motI-mot2. Tout constat empirique établissant une relation entre la fréquence des collocations motimot2 et l'apprentissage des liaisons correctes joue donc en faveur de la conception exemplariste. C'est notre seconde prédiction.

Nous allons maintenant présenter les trois études de cas. Pour chacune d'elles, nous soulignerons les principales tendances et nous nous demanderons dans quelle mesure elles confirment, précisent, contredisent les repères développementaux établis à partir de tâches expérimentales. Parmi ces tendances, nous approfondirons deux résultats susceptibles de trancher entre les prédictions des deux modèles présentés en introduction.

3 Cinq OBSERVATIONS TRANSVERSALES ENTRE 3 ET I I ANS

\subsection{Méthode et données}

La première étude est fondée sur les contextes de liaison produits par cinq enfants âgés de 3 à II ans, enregistrés lors d'interactions au domicile familial. Tous vivent dans la région Rhône-Alpes. Le tableau I présente les enfants, la tranche d'âge des recueils et la profession des parents. Il précise également les conditions d'enregistrement et le lien entre les enquêtrices et les familles. Les interactions observées impliquent l'enfant et une jeune femme qui assure sa garde, l'aide à faire ses devoirs ou est amie de la famille, excepté pour la fillette D., enregistrée en interaction avec son père ou sa mère, hors de la présence de l'enquêtrice ${ }^{3}$. Il faut noter également que le garçon B. et la fillette C. sont jumeaux et participent donc à des environnements langagiers familiaux en partie identiques.

Le tableau 2 présente les pourcentages individuels de liaisons réalisées conformément à la cible adulte et, dans les trois dernières lignes, l'incidence des deux types d'erreurs relevés dans les contextes de liaison: les substitutions (un ours prononcé [õezurs], où /z/ remplace le $/ \mathrm{n} /$ attendu) et les omissions en contexte obligatoire (des ours prononcé [deurs] sans liaison).

Nous avons distingué les contextes obligatoires des contextes facultatifs en adoptant la répartition de Booij et de Jong (I987). Parce qu'ils observent les usages

3 Nous remercions Bénédicte Basset, Gaëlle Grégoire, Sandrine Malosse, Ouahiba Meradji et Sylvie Rosiak pour leur contribution au recueil et au traitement des données transversales. 
Tableau I. Cinq études de cas: sujets et situations d'enregistrement

\begin{tabular}{|c|c|c|c|c|}
\hline $\begin{array}{l}\text { Code identifiant } \\
\text { l'enfant et sexe }\end{array}$ & $\begin{array}{l}\text { Fourchette d'âge } \\
\text { des enregistrements }\end{array}$ & Profession des parents & $\begin{array}{l}\text { Lieu et situations de } \\
\text { recueil }\end{array}$ & Lien avec l'enquêtrice \\
\hline A. (fille) & $3 ; 4$ à $4 ; 0$ & Petits commerçants & $\begin{array}{l}\text { Domicile de la famille } \\
\text { Conversations entre } \\
\text { l'enquêtrice et la fillette } \\
\text { lors de repas, de jeux } \\
\text { ou de bains }\end{array}$ & $\begin{array}{l}\text { L'enquêtrice garde la } \\
\text { fillette à domicile }\end{array}$ \\
\hline B. (garçon, jumeau de C.) & $5 ; 8$ à $5 ; \mathrm{IO}$ & Père: technicien $(\mathrm{Bac}+2)$ & Domicile de la famille & Les enquêtrices sont \\
\hline C. (fille, jumelle de B.) & $5 ; 8$ à $5 ; 10$ & Mère: infirmière & $\begin{array}{l}\text { Conversations entre les } \\
\text { enfants et les } \\
\text { enquêtrices au cours de } \\
\text { jeux ou de repas }\end{array}$ & $\begin{array}{l}\text { voisines et amies de la } \\
\text { famille }\end{array}$ \\
\hline D. (fille) & 6; IO à 7;0 & $\begin{array}{l}\text { Père: médecin } \\
\text { Mère: au foyer }\end{array}$ & $\begin{array}{l}\text { Domicile de la famille } \\
\text { Conversations entre la } \\
\text { fillette et un des deux } \\
\text { parents }\end{array}$ & $\begin{array}{l}\text { Magnétophone confié aux } \\
\text { parents, amis de } \\
\text { l'enquêtrice }\end{array}$ \\
\hline E. (fille) & IO;I I à I I;4 & $\begin{array}{l}\text { Père: représentant } \\
\text { Mère: aide ménagère }\end{array}$ & $\begin{array}{l}\text { Domicile de la famille } \\
\text { Conversations entre la } \\
\text { fillette et l'enquêtrice } \\
\text { (soutien scolaire, } \\
\text { conversations de } \\
\text { sociabilité) }\end{array}$ & $\begin{array}{l}\text { L'enquêtrice aide la fillette } \\
\text { à faire ses devoirs }\end{array}$ \\
\hline
\end{tabular}


Tableau 2. Cinq études de cas: liaisons correctes et erreurs (NB - Les pourcentages ne sont calculés que pour un effectif supérieur à 10 occurrences)

\begin{tabular}{|c|c|c|c|c|c|}
\hline Sujet et âge approximatif $\rightarrow$ & $\begin{array}{l}\text { A. } \\
(3-4 \\
\text { ans) }\end{array}$ & $\begin{array}{l}\text { B. } \\
(5-6 \\
\text { ans) }\end{array}$ & $\begin{array}{l}\text { C. } \\
(5-6 \\
\text { ans) }\end{array}$ & $\begin{array}{l}\text { D. } \\
\text { (6-7 } \\
\text { ans) }\end{array}$ & $\begin{array}{l}\text { E. } \\
\text { (IO-II } \\
\text { ans) }\end{array}$ \\
\hline \multirow[t]{2}{*}{$\begin{array}{l}\text { Liaisons obligatoires justes } \\
\text { a) après un déterminant (les_ours) }\end{array}$} & $77 \%$ & $91 \%$ & $97 \%$ & $97 \%$ & $100 \%$ \\
\hline & I I9/I 54 & $6 \mathrm{I} / 67$ & $7 \mathrm{I} / 73$ & $7 \mathrm{I} / 73$ & $\mathrm{IOO} / \mathrm{IOO}$ \\
\hline \multirow{2}{*}{$\begin{array}{l}\text { b) après un pronom clitique } \\
\text { préverbal (j'en_ai, ils_ont) }\end{array}$} & $91 \%$ & $100 \%$ & $100 \%$ & $100 \%$ & $99 \%$ \\
\hline & I 3 I / I 44 & $\mathrm{I} 4 \mathrm{O} / \mathrm{I} 4 \mathrm{O}$ & $205 / 205$ & $205 / 205$ & $263 / 264$ \\
\hline \multirow{2}{*}{$\begin{array}{l}\text { c) dans une expression figée } \\
\text { (tout_à coup) }\end{array}$} & - & $100 \%$ & - & $100 \%$ & $100 \%$ \\
\hline & $\mathrm{I} / \mathrm{I}$ & $\mathrm{I} 3 / \mathrm{I} 3$ & - & I I / I I & $45 / 45$ \\
\hline $\begin{array}{l}\text { d) entre verbe et clitique post-verbal } \\
\text { (prenez_en!vient_il ?) }\end{array}$ & - & $\mathrm{I} / \mathrm{I}$ & - & $\begin{array}{l}- \\
-\end{array}$ & - \\
\hline $\begin{array}{l}\text { Total liaisons obligatoires } \\
\text { justes }\end{array}$ & $\begin{array}{l}84 \% \\
25 I / 299\end{array}$ & $\begin{array}{l}97 \% \\
215 / 221\end{array}$ & $\begin{array}{l}99 \% \\
\text { I87/I89 }\end{array}$ & $\begin{array}{l}99 \% \\
287 / 289\end{array}$ & $\begin{array}{l}99 \% \\
408 / 409\end{array}$ \\
\hline \multicolumn{6}{|l|}{ Liaisons facultatives justes } \\
\hline \multirow{2}{*}{$\begin{array}{l}\text { e) après un adjectif pré-nominal } \\
\text { (un petit_ours) }\end{array}$} & $48 \%$ & - & - & - & - \\
\hline & I I $/ 23$ & $3 / 4$ & $6 / 8$ & $4 / 7$ & $3 / 10$ \\
\hline \multirow{2}{*}{$\begin{array}{l}\text { f) après un nom pluriel } \\
\text { (des enfants_idiots) }\end{array}$} & $2 \%$ & $18 \%$ & - & $0 \%$ & $0 \%$ \\
\hline & $\mathrm{I} / 58$ & $2 /$ I I & $2 / 8$ & $\mathrm{O} / \mathrm{I} 3$ & $\mathrm{O} / \mathrm{I} 7$ \\
\hline \multirow{2}{*}{$\begin{array}{l}\text { g) après une forme du verbe avoir } \\
\text { (ils ont_un...) }\end{array}$} & - & $0 \%$ & - & $0 \%$ & $0 \%$ \\
\hline & $0 / 7$ & $0 / 15$ & $0 / 7$ & $0 / 50$ & $0 / 28$ \\
\hline \multirow{2}{*}{$\begin{array}{l}\text { h) après une forme du verbe être } \\
\text { (ils sont_ici) }\end{array}$} & $0 \%$ & $5 \%$ & $0 \%$ & $\mathbf{I} 2 \%$ & $20 \%$ \\
\hline & $0 / 49$ & $4 / 76$ & $0 / 50$ & Io/84 & $39 / 198$ \\
\hline \multirow{2}{*}{$\begin{array}{l}\text { i) après une forme d'un autre verbe } \\
\text { (il vient_aussi) }\end{array}$} & $1 \%$ & $2 \%$ & $1 \%$ & $0 \%$ & $5 \%$ \\
\hline & I/94 & 2/I06 & $\mathrm{I} / 78$ & O/I I 8 & $\mathrm{IO} / 2 \mathrm{I} 4$ \\
\hline \multirow{2}{*}{$\begin{array}{l}\text { j) après mot invariable } \\
\text { (en_Espagne, quand_un...) }\end{array}$} & $3 \%$ & $7 \%$ & $26 \%$ & $24 \%$ & $12 \%$ \\
\hline & $3 / 99$ & $8 / \mathrm{I} 2 \mathrm{I}$ & I9/73 & $35 / 146$ & $27 / 220$ \\
\hline $\begin{array}{l}\text { Total liaisons facultatives } \\
\text { justes }\end{array}$ & $\begin{array}{l}5 \% \\
16 / 330\end{array}$ & $\begin{array}{l}6 \% \\
19 / 333\end{array}$ & $\begin{array}{l}12 \% \\
28 / 224\end{array}$ & $\begin{array}{l}12 \% \\
49 / 418\end{array}$ & $\begin{array}{l}12 \% \\
79 / 687\end{array}$ \\
\hline $\begin{array}{l}\text { Erreurs de substitution en } \\
\text { contexte de liaison } \\
\text { obligatoire } \\
\text { (un ours produit [œẽzurs] où } \\
\text { /z/ remplace le /n/ } \\
\text { attendu) }\end{array}$ & $\begin{array}{l}\mathbf{5} \% \\
\text { I } 6 / 299\end{array}$ & $\begin{array}{l}0 \% \\
0 / 22 I\end{array}$ & $\begin{array}{l}0 \% \\
0 / 189\end{array}$ & $\begin{array}{l}\mathbf{0} \% \\
0 / 289\end{array}$ & $\begin{array}{l}0 \% \\
0 / 409\end{array}$ \\
\hline $\begin{array}{l}\text { Erreurs de substitution en } \\
\text { contexte de liaison } \\
\text { facultative } \\
\text { (je suis allé produit [ [ yitale] } \\
\text { où /t/ remplace le /z/ } \\
\text { attendu) }\end{array}$ & $\begin{array}{l}3 \% \\
9 / 330\end{array}$ & $\begin{array}{l}0.6 \% \\
2 / 333\end{array}$ & $\begin{array}{l}0 \% \\
0 / 224\end{array}$ & $\begin{array}{l}0 \% \\
0 / 4 \mathrm{I} 8\end{array}$ & $\begin{array}{l}\mathbf{I . 5} \% \\
10 / 687\end{array}$ \\
\hline $\begin{array}{l}\text { Erreurs d'omission en } \\
\text { contexte de liaison } \\
\text { obligatoire } \\
\text { (des ours prononcé [deurs] } \\
\text { sans aucune liaison) }\end{array}$ & $\begin{array}{l}\mathbf{I} \mathbf{\%} \% \\
32 / 299\end{array}$ & $\begin{array}{l}3 \% \\
6 / 22 I\end{array}$ & $\begin{array}{l}\mathbf{I} \% \\
2 / \mathrm{I} 89\end{array}$ & $\begin{array}{l}\mathbf{I} \% \\
4 / 289\end{array}$ & $\begin{array}{l}0.2 \% \\
\mathrm{I} / 409\end{array}$ \\
\hline
\end{tabular}


réels des adultes dans plusieurs corpus, ces auteurs évitent la tentation normative de définir a priori l'obligation de liaison. Ils constatent que, chez l'adulte, la liaison n'est catégorique que dans quatre contextes: après un déterminant, après un pronom pré-verbal, entre un verbe et un pronom qu'il régit (prenez-en!), dans certaines expressions figées (tout à coup).

\subsection{Les liaisons obligatoires}

Les liaisons obligatoires semblent bien maîtrisées à $3-4$ ans $(84 \%)$ et elles sont acquises totalement à partir de 5-6 ans. Les valeurs de $97 \%$ et $98 \%$ de réussite chez B. et C. sont identiques à la performance adulte. En effet, on trouve chez le père des jumeaux, enregistré en entretien (Meradji et Grégoire, 200I), deux cas sporadiques de non liaison entre clitique et verbe, ce qui ramène son taux global de réalisation à $98 \%$ (I IO/I I 2). C'est donc entre 4 et 5 ans que semble s'établir une maitrise des liaisons obligatoires semblable à celle de l'adulte. Cette conclusion confirme les résultats établis pour les séquences déterminant-nom lors de tâches expérimentales proposées à de très grands échantillons d'enfants de 2 à 6 ans (Dugua, 2002; Nardy, 2003). L'éventail des contextes recueillis dans les cinq études de cas permet toutefois de préciser que la liaison obligatoire après un pronom clitique (9I\% dès $3-4$ ans, I00\% ou presque ensuite) semble maitrisée avant celle qui suit un déterminant (77\% à 3-4 ans et 9I\% chez B. à 5-6 ans, I00\% ou presque ensuite). On constate dans le tableau 2 que les contextes Après un pronom clitique sont plus nombreux chez quatre des cinq enfants que les contextes Après un déterminant. Cette différence subsiste d'ailleurs chez les parents des jumeaux B. et C.: 70 contextes pronominaux pour le père et I 57 pour la mère contre respectivement 34 et 57 contextes suivant un déterminant (Meradji et Grégoire, 200I). Si on admet que la fréquence est un élément central de l'apprentissage des liaisons obligatoires correctes, alors il est attendu que les enfants apprennent plus vite les liaisons qui suivent des mots qu'ils entendent souvent. Ainsi, la fréquence élevée des mots I favoriserait non seulement la réalisation des liaisons facultatives chez l'adulte (Ågren, I973; Booij et de Jong, I987), mais également l'apprentissage des liaisons obligatoires chez l'enfant.

Plus généralement, l'hétérogénéité développementale qui subsiste à 3 ans offre l'occasion d'étendre, aux liaisons obligatoires, la recherche des différents effets de fréquence, dont l'existence chez l'adulte est bien connue mais nécessairement limitée aux liaisons optionnelles. En réanalysant les données de Ågren (I973), Bybee (200I, 2005) suggère que la fréquence élevée d'une construction particulière de type semi-auxiliaire + infinitif (falloir + être, devoir + avoir, etc.) favorise la réalisation de la liaison facultative entre les deux éléments. Alexander (2004) teste systématiquement l'hypothèse de Bybee. A travers une tâche expérimentale, dix sujets produisent des séquences moti-mot2 particulières (hommes heureux, très ancien) dont la fréquence a été calculée dans le corpus électronique Frantext. Dans les contextes facultatifs nom + adjectif et adverbe + adjectif, la réalisation des liaisons facultatives est plus importante dans les collocations les plus fréquentes. 
Tableau 3. Liaisons obligatoires après clitique ou déterminant à 3 ans: effet de la fréquence du mot1 et de la fréquence des collocations mot1-mot2

\begin{tabular}{|c|c|c|c|c|c|c|c|}
\hline \multirow[t]{2}{*}{$\begin{array}{l}\text { I- } \\
\text { Mots I }\end{array}$} & \multicolumn{2}{|c|}{$\begin{array}{l}2 \text { - Pourcentage } \\
\text { de liaisons justes }\end{array}$} & \multicolumn{2}{|c|}{$\begin{array}{l}3-\text { Nombre } \\
\text { d'occurrences du } \\
\text { motI }\end{array}$} & \multirow[t]{2}{*}{$\begin{array}{l}- \text { Nombre de } \\
\text { mots2 différents } \\
\text { après le mot I }\end{array}$} & \multicolumn{2}{|c|}{$\begin{array}{l}5-\text { Rapport du } \\
\text { nombre } \\
\text { d'occurrences de } \\
\text { motI par rapport } \\
\text { au nombre de } \\
\text { mots2 différents }\end{array}$} \\
\hline & valeur & ordre & valeur & ordre & & valeur & ordre \\
\hline on & 28,6 & I & 7 & I & 4 & $\mathrm{I}, 75$ & I \\
\hline elles & 62,5 & 2 & 8 & 2 & 4 & 2,00 & 2 \\
\hline des & $7 \mathrm{I}, 8$ & 3 & 39 & 5 & I6 & 2,44 & 3 \\
\hline les & 80,7 & 4 & 57 & 7 & 20 & 2,85 & 4 \\
\hline un & $8 \mathrm{I}, 6$ & 5 & 49 & 6 & I2 & 4,08 & 6 \\
\hline ils & 88,2 & 6 & I7 & 4 & 4 & 4,25 & 7 \\
\hline ses & 90,9 & 7 & I I & 3 & 3 & 3,67 & 5 \\
\hline en & $97, \mathrm{I}$ & 8 & IO4 & 8 & 7 & $\mathrm{I} 4,86$ & 8 \\
\hline
\end{tabular}

Nous allons donc examiner l'impact éventuel de la fréquence des séquences motI-mot2 sur la capacité enfantine à réaliser des liaisons obligatoires justes. Cet examen nous offre la possibilité de confronter les deux modèles développementaux exposés dans la section 2 (voir la seconde prédiction, section 2.3). Comme nous l'avons exposé précédemment, seul le modèle exemplariste et basé sur l'usage (Chevrot, Dugua et Fayol, 2005) prédit que la liaison obligatoire est apprise plus vite dans les séquences moti-mot2 les plus fréquentes. En nous limitant aux liaisons suivant un déterminant ou un clitique, qui offrent un matériau suffisant chez l'enfant A. de 3 ans, nous avons calculé plusieurs indices de fréquence présentés dans le tableau 3. Dans la première colonne apparaissent les déterminants et les clitiques dont la fréquence d'occurrence est supérieure à 6 dans le corpus de l'enfant A. Ces 8 mots I sont rangés dans l'ordre croissant des scores de liaisons justes qui apparaissent dans la seconde colonne. Dans la troisième colonne, on trouve le nombre d'occurrences de chacun de ces déterminants et clitiques dans l'enregistrement de A. Dans la quatrième colonne apparaît le nombre de mots2 différentes trouvées dans l'enregistrement de A. après chacun des déterminants et clitiques. Par exemple, le clitique en est suivi de 7 unités: a, achète, acheter, ai, as, aura, on. Dans la dernière colonne, nous avons calculé le rapport des valeurs de la colonne 3 (nombre d'occurrences du moti) aux valeurs de la colonne 4 (nombre de mots 2 différents après un motI). Ce rapport estime le nombre moyen de séquences motImot2 dans lesquelles apparait chaque moti en contexte de liaison. Par exemple, puisque le clitique elles comptabilise 8 occurrences précédant quatre mots2, chacun des contextes lexicaux de liaison motI-mot2 où le clitique est inclus apparaît en moyenne deux fois. Plus ce rapport est grand et plus un moti apparait dans des contextes moti-mot2 fréquents. 
Le classement des mots I selon les pourcentages de liaisons justes (colonne 2) est quasi identique à leur classement selon le rapport de la colonne 5 , à l'exception du possessif ses. Plus un clitique ou un déterminant est employé dans des contextes de liaisons moti-mot2 fréquents, plus la liaison est réalisée juste par l'enfant de 3 ans. Le calcul d'un coefficient de corrélation par rang entérine cette tendance (Rho de Spearman $=0.93, \mathrm{p}=$ o.oI4) alors que le pourcentage de justes (colonne 2) ne corrèle significativement ni avec le nombre d'occurrences du moti (colonne 3, $\mathrm{p}=$ o.I I), ni avec le nombre de mots2 (colonne $4, \mathrm{p}=0.75$ ). Ainsi, comme le prédit le modèle exemplariste et basé sur l'usage, un enfant de 3 ans réussit mieux les liaisons obligatoires dans des séquences lexicales moti-mot2 qu'il produit souvent et dont on peut penser qu'elles sont souvent entendues.

\subsection{Les liaisons facultatives}

L'évolution développementale des liaisons facultatives est différente du patron d'acquisittion des liaisons obligatoires. On voit dans le tableau 2 qu'elles sont réalisées dans $5 \%$ des cas à $3-4$ ans. A 5-6 ans apparait une variation interindividuelle entre B. et C., bien qu'ils soient jumeaux et participent à des environnements langagiers en partie identiques. Le garçon B. obtient un taux de réalisation comparable à celui de l'enfant de 3-4 ans (6\%) tandis que le score de sa jumelle C. vaut I $2 \%$, valeur qui reste identique chez les enfants de 6-7 ans et IO-I I ans et qui pourrait être la performance définitive en situation non formelle. En effet, chez I6 adultes et adolescents d'origines sociales variées enregistrés dans des situations familières, la liaison facultative est réalisée dans I0\% des cas seulement (Ahmad, I993). L'usage des liaisons optionnelles en situation familière pourrait donc être en place vers 6-7 ans, avec une variation interindividuelle vers $5-6$ ans.

Ces résultats précisent les études réalisées à l'aide de tâches expérimentales limitées au contexte adjectif-nom, considéré comme facultatif puisque les adultes n’y réalisent pas I00\% des occurrences (Booij et de Jong, I987). Premièrement, les pourcentages de réalisation obtenus à partir du seul contexte adjectif-nom (de I I \% à $2-3$ ans à 33\% à 5-6 ans après les adjectifs petit et gros, Dugua, 2002) sont supérieurs aux valeurs calculées sur l'ensemble des contextes de liaison facultative (de $5 \%$ à I $2 \%$, tableau 2). Dans les environnements où la liaison reste variable, son acquisition en position post-adjectivale à l'intérieur du groupe nominal semble donc jouer un rôle précurseur, comme en témoigne la valeur de $48 \%$ attestée dès $3-4$ ans chez A. Deuxièmement, l'étude de corpus confirme que le développement des liaisons optionnelles s'effectue sur une longue période. Des progrès notables après un mot invariable ne sont pas enregistrés avant $5-6$ ans $(26 \%$ chez C.) et pas avant $6-7$ ans à la suite des formes du verbe être (I $2 \%$ chez D.). D'autres évolutions dans des registres plus contrôlés sont d'ailleurs attendues après IO-I I ans, puisque le taux global de I $2 \%$ de l'enfant E. est loin d'atteindre les $79 \%$ constatés chez des adultes « cultivés » enregistrés en situation formelle (Ahmad, I993). 


\subsection{Les erreurs}

L'observation des erreurs confirme également les études expérimentales menées entre 2 et 6 ans (Chevrot et Fayol, 200 I; Dugua, 2002). Les erreurs de substitution sont très fréquentes à 3 ans puis disparaissent à partir de 5 ans, alors que les omissions en contexte obligatoire semblent persister davantage. L'étude de corpus suggère un fait nouveau: la réapparition tardive d'erreurs de substitution dans les contextes facultatifs ( $0 \%$ de 5 à 7 ans puis I. $5 \%$ à IO-I I ans) pourrait accompagner les progrès tardifs dans la réalisation des liaisons correspondantes. En effet, les Io cas d'erreurs recensés à IO-I I ans concernent tous des contextes qui pilotent l'acquisition des productions: après une forme du verbe être (tu es allé produit [tyetale]) et après un mot invariable (Combien ça fait en heures produit [kõbjẽsafeãzœr]).

DEUX SUIVIS LONGITUdinAUX ENTRE 40 ET 50 MOIS

\subsection{Méthode et données}

Parce que la méthode transversale compare des individus différents d'âges différents, elle est susceptible de confondre des différences interindividuelles avec des évolutions développementales. Afin de distinguer ces deux facteurs, nous avons réalisé le suivi longitudinal de deux jeunes garçons, F. et G., domiciliés en Ardèche et enregistrés individuellement entre 40 et 50 mois. Ces enregistrements ont eu lieu lors de cinq sessions échelonnées tous les deux mois environ, dans une salle calme des écoles fréquentées par les enfants. Pendant ces sessions, qui duraient une trentaine de minutes, chaque enfant interagissait avec l'enquêteur à l'occasion de jeux (construction, dînette), de récits sur leur emploi du temps et de dialogues autour de livres d'images. L'enquêteur a fréquenté la classe des enfants et les a rencontrés individuellement plusieurs fois avant le début des enregistrements.

Les milieux sociaux des deux enfants sont contrastés. Le père de G. est technicien supérieur, sa mère cadre commerciale et tous deux ont fait deux années d'études dans l'enseignement supérieur. Le père de F. est ouvrier sans qualification dans le secteur du bâtiment et sa mère s'occupe du foyer. Tous deux ont interrompu leur scolarité vers l'âge de I 4 ans.

La période d'enregistrement de Io mois a été divisée en deux sous-périodes: 40 à 47 mois et 48 à 50 mois. Celles-ci sont délimitées de façon à équilibrer le nombre de contextes de liaison recueillis dans chacun des intervalles, la longueur des énoncés enfantins augmentant avec l'âge. Dans le tableau 4, figurent les taux d'erreurs et de réalisation des liaisons facultatives et obligatoires des deux enfants dans chacune des tranches biographiques.

\subsection{Commentaires: liaisons obligatoires, liaisons facultatives et erreurs}

Les réalisations des liaisons obligatoires progressent entre les deux tranches d'âge pour les deux enfants. Dans les deux périodes, les taux de l'enfant G., issu de parents ayant fréquenté l'enseignement supérieur, dépassent les taux de l'enfant F, dont les 
Tableau 4. Evolution longitudinale des liaisons obligatoires et facultatives et des erreurs chez deux enfants entre 40 et 50 mois

\begin{tabular}{|c|c|c|c|}
\hline & & $\begin{array}{l}\text { Tranche I } \\
40-47 \text { mois }\end{array}$ & $\begin{array}{l}\text { Tranche } 2 \\
48-50 \text { mois }\end{array}$ \\
\hline \multirow[t]{4}{*}{ Liaisons obligatoires justes } & Enfant $\mathrm{F}$. & $76 \%$ & $88 \%$ \\
\hline & & I30/I 70 & I $52 / 172$ \\
\hline & Enfant G. & $89 \%$ & $99 \%$ \\
\hline & & $\mathrm{I} 45 / \mathrm{I} 62$ & $\mathrm{I} 7 \mathrm{I} / \mathrm{I} 72$ \\
\hline \multirow[t]{4}{*}{ Liaisons facultatives justes } & Enfant $\mathrm{F}$. & $5 \%$ & $6 \%$ \\
\hline & & $4 / 83$ & 4/66 \\
\hline & Enfant G. & $7 \%$ & $21 \%$ \\
\hline & & $9 / \mathrm{I} 38$ & 22/IO4 \\
\hline \multirow{4}{*}{$\begin{array}{l}\text { Erreurs de substitution en } \\
\text { contexte de liaison obligatoire } \\
\text { (un ours produit [õzurs] où / } \mathrm{z} / \\
\text { remplace le } / \mathrm{n} / \text { attendu) }\end{array}$} & Enfant $\mathrm{F}$. & $17 \%$ & $9 \%$ \\
\hline & & $29 / 170$ & I6/I 72 \\
\hline & Enfant G. & $8 \%$ & $0.5 \%$ \\
\hline & & I3/I62 & $\mathrm{I} / \mathrm{I} 72$ \\
\hline \multirow{4}{*}{$\begin{array}{l}\text { Erreurs de substitution en } \\
\text { contexte de liaison facultative } \\
\text { (je suis allé produit }\left[\int \text { yitale] où }\right. \\
\text { /t/ remplace le } / \mathrm{z} / \text { attendu) }\end{array}$} & Enfant $\mathrm{F}$. & $22 \%$ & $27 \%$ \\
\hline & & I $8 / 83$ & I $8 / 66$ \\
\hline & Enfant G. & $20 \%$ & $28 \%$ \\
\hline & & $28 /$ I 38 & 29/IO4 \\
\hline \multirow{3}{*}{$\begin{array}{l}\text { Erreurs d'omission en contexte } \\
\text { de liaison obligatoire } \\
\text { (des ours prononcé [deurs] } \\
\text { sans aucune liaison) }\end{array}$} & Enfant F. & $6 \%$ & $2 \%$ \\
\hline & Enfant G. & $\begin{array}{l}\text { I I / I } 70 \\
\mathbf{2} \%\end{array}$ & $\begin{array}{l}4 / \mathrm{I} 72 \\
0 \%\end{array}$ \\
\hline & & $4 / 162$ & $\mathrm{O} / \mathrm{I} 72$ \\
\hline
\end{tabular}

parents sont peu scolarisés. Toutefois, cette différence va nécessairement se resserrer lorsque les enfants avanceront en âge, puisque le score de G. a quasiment atteint le maximum de Io०\% dans la tranche d'âge 2 alors que celui de F. peut encore progresser.

Les liaisons facultatives présentent un profil différent. Seul l'enfant G. progresse notablement entre les deux tranches d'âge en multipliant par trois le pourcentage de réalisation, qui passe de $7 \%$ à $2 \mathrm{I} \%$. Le score de l'enfant F. reste proche de $5 \%$ dans les deux périodes. De ce fait, la différence entre les deux enfants s'accroît avec l'âge.

Enfin, tous les types d'erreurs diminuent entre la première tranche d'âge et la seconde, sauf les erreurs de substitution dans les contextes de liaison facultative, dont l'incidence tend au contraire à augmenter.

Ces résultats longitudinaux confirment et précisent l'étude transversale. Les liaisons obligatoires atteignent effectivement un taux proche de IOo\% dans la cinquième année, mais la vitesse de progression varie selon les enfants. L'évolution des liaisons facultatives donne également lieu à de fortes variations entre les deux enfants. Puisque F. et G. sont issus de milieux contrastés, on peut se demander comment ces différences entre eux sont liées à leur origine sociale.

Les différences interindividuelles affectant les liaisons facultatives trouveraient leur origine dans l'apprentissage culturel de normes d'usage et seraient de nature 
sociolinguistique. Les deux enfants apprennent progressivement le sociolecte environnant et cet apprentissage crée des distinctions qui s'accentuent avec l'âge. Au contraire, les écarts concernant les liaisons obligatoires résulteraient directement de la mise en œuvre du mécanisme d'acquisition. En effet, dans le scénario basé sur l'usage présenté en introduction (voir section 2.I), la seconde étape consiste à mémoriser les relations entre un moti particulier et la variante adéquate du mot2. Or on sait que les enfants de parents à statut social élevé entendent davantage de parole adulte (Hoff, 2002). Davantage de parole adulte entendue, c'est davantage d'occasions de rencontrer des séquences moti-mot2 bien formées. Le modèle basé sur l'usage prédit donc un apprentissage des liaisons obligatoires plus rapide chez les enfants de familles à statut social élevé, même si aucune différence sociolinguistique n'oppose les adultes quant à l'usage de ce type de liaisons. Lorsque vient l'étape 3 des généralisations, ces différences liées à la quantité d'input disparaissent.

Ces résultats longitudinaux acquis en situation naturelle confirment absolument les données expérimentales recueillies par Nardy (2003) auprès de I 89 enfants de 2 à 6 ans. Dans le contexte obligatoire déterminant + nom, elle observe que les pourcentages moyens de liaisons justes sont significativement plus élevés pour le milieu défini comme «cadre » que pour le milieu défini comme « ouvrier ». Cette différence subsiste à tous les âges mais s'amenuise progressivement. Dans le contexte variable adjectif + nom, la différence entre les deux milieux augmente progressivement avec l'âge et ne devient statistiquement significative qu'entre 6I et 72 mois. Ainsi, les différences résultant de l'apprentissage de sociolectes qualitativement différents s'accroissent avec l'âge alors que celles qui résultent d'inputs quantitativement différents diminuent puis disparaissent.

\section{Etude d'un Corpus d'erreurs et efFets de Frequence}

Parmi les données présentées jusqu'ici, un seul résultat tranche en faveur d'une des prédictions découlant du modèle basé sur l'usage: la relation à 3 ans entre le taux de liaisons correctes après clitiques et déterminants et la fréquence des combinaisons moti-mot2 dans lesquelles rentrent ces clitiques et ces déterminants. Nous allons maintenant examiner l'autre prédiction (cf. section 2.3, première prédiction): l'éventualité d'un lien entre la nature phonétique des liaisons enfantines $(/ \mathrm{n} /, / \mathrm{z} / \mathrm{ou} / \mathrm{t} /)$ et le nom qui suit.

Un recueil d'erreurs de liaison obtenu en situation naturelle permet de tester cette prédiction. Certaines de ces erreurs surviennent devant des mots 2 dont le sens induit plutôt un usage au pluriel ou plutôt un usage au singulier. Pour des raisons référentielles évidentes, l'enfant devrait entendre le mot2 habit plus souvent au pluriel (Range tes habits!) alors que le mot2 arc-en-ciel devrait être entendu majoritairement au singulier (il y a un arc-en-ciel.). L'enfant devrait donc entendre plus souvent habit précédé de la liaison /z/ et arc-en-ciel précédé de la liaison /n/. Selon le modèle basé sur l'usage, les jeunes enfants devraient produire les mots orientés vers le pluriel en privilégiant l'exemplaire commençant par /z/. En effet, cet exemplaire étant plus souvent perçu dans l'input, il a plus de chances d'être 
segmenté et plus de chances d'être renforcé. Il sera donc plus disponible dans le lexique enfantin et émergera plus facilement dans les erreurs. De ce fait, devant un mot comme habit, l'erreur en /z/ (un habit produit [õezabi]) devrait être plus fréquente que dans un mot comme arc-en-ciel qui est orienté vers le singulier. Notre travail a donc d'abord consisté à trouver des indices de l'orientation pluriel ou singulier des mots2, d'une part à travers l'intuition de locuteurs adultes et d'autre part dans un corpus de textes. Ensuite, nous avons observé le lien entre cette orientation et les erreurs enfantines relevées en situation naturelle.

\subsection{Méthodes et données}

Les erreurs ont été recueillies dans le cadre familial par le père de la fillette $\mathrm{S}$. L'observateur notait sur un carnet les erreurs qu'il entendait, en transcrivant pour chaque relevé l'énoncé de l'enfant ainsi que la date précise. Cette saisie se faisait en dehors de la présence de la fillette qui n'a été informée qu'ultérieurement de ce recueil. Cette notation a débuté quand S. avait 2 ans I mois et elle s'est terminée lorsqu'elle a atteint 6 ans 4 mois. Au total, 898 erreurs ont été notées et analysées. D'une part, il s'agit de 545 ajouts dans lesquels une consonne de liaison ou le /1/ résultant de l'élision est inséré dans un contexte où la cible adulte les interdit (papa aigle est produit [papanegl]). D'autre part, il s'agit de 353 substitutions dans lesquelles une consonne de liaison attendue est remplacée par une liaison fautive ou le /l/ de l'élision (grand éclair est produit [grãnekler], un avion est produit [õelavjõ]).

Pour mener à bien la présente analyse, nous n'avons retenu que les 437 occurrences d'erreurs apparaissant devant un nom masculin. L'exclusion des noms féminins est motivée par le fait que ces derniers ne sont pas précédés d'une liaison $/ \mathrm{n} /$ au singulier mais $\mathrm{du} / \mathrm{n} /$ enchainé entre des déterminants tels que une ou aucune et le mot suivant. Enfin, parmi les noms masculins, nous avons également exclu ceux dont l'effectif était inférieur ou égal à 5 ainsi que trois noms dont la forme au singulier est différente de la forme au pluriel (œuf/ œufs, œil/yeux, animal/animaux). Ces trois noms posent des questions spécifiques de contingence entre les formes de mot et les liaisons $/ \mathrm{z} /$ et $/ \mathrm{n} /$. Nous les traiterons à part.

Ces différentes restrictions aboutissent à la sélection de I 3 noms masculins apparaissant au moins dans 6 erreurs. Ils sont listés dans la colonne I du tableau 5 , où ils sont suivis des types d'erreur qui les affectent (colonnes 2 à 5 ). Ainsi, parmi les erreurs de S., le mot ami apparait au total dans I 2 liaisons erronées (colonne 6): une erreur en /n/ (ses amis produit [senami]), onze en /z/ (autre ami produit [otzami]), aucune en $/ \mathrm{t} / \mathrm{ni}$ en $/ 1 /$. Le rapport calculé dans la colonne 7 estime la tendance d'un mot à provoquer les erreurs en $/ \mathrm{z} /$ plutôt que les erreurs en /n/. Dans le cas de ami, ce rapport entre le nombre d'erreurs en /z/ et le total des erreurs en /z/ et des erreurs en $/ \mathrm{n} /$ vaut 9I,67\% (I I/I + I I). Le mot ami tend donc à provoquer les erreurs en /z/. Inversement, pour anorak, arc-en-ciel, éléphant, escalier ${ }^{4}$ et ours, ce

${ }^{4}$ Dans le français quotidien, le mot escalier est utilisé au singulier. 
Tableau 5. Erreurs entre 2 et 6 ans chez S. et orientation singulier/pluriel des noms

\begin{tabular}{|c|c|c|c|c|c|c|c|c|}
\hline \multirow[t]{2}{*}{ I Noms } & \multicolumn{4}{|c|}{ Types d'erreurs } & \multirow{2}{*}{$\begin{array}{l}6 \text { Total } \\
\text { erreurs }\end{array}$} & \multirow{2}{*}{$\begin{array}{l}7 \text { Rapport } \\
\mathrm{z} / \mathrm{z}+\mathrm{n} \text { dans } \\
\text { les erreurs } \\
\text { et ordre }\end{array}$} & \multirow{2}{*}{$\begin{array}{l}8 \text { Rapport } \\
\mathrm{pl} / \mathrm{pl}+\mathrm{sg} \\
\text { dans le test } \\
\text { d'intuition } \\
\text { et ordre }\end{array}$} & \multirow{2}{*}{$\begin{array}{l}\text { 9 Rapport } \\
\mathrm{pl} / \mathrm{pl}+\mathrm{sg} \\
\text { dans } \\
\text { Frantext et } \\
\text { ordre }\end{array}$} \\
\hline & $\begin{array}{l}2-\mathrm{en} \\
/ 1 /\end{array}$ & $\begin{array}{l}3-\text { en } \\
/ \mathrm{n} /\end{array}$ & $\begin{array}{l}4-\mathrm{en} \\
/ \mathrm{t} /\end{array}$ & $\begin{array}{l}5-\text { en } \\
\mid z /\end{array}$ & & & & \\
\hline Anorak & $\mathrm{O}$ & 6 & o & o & 6 & $\mathrm{O}, \mathrm{O} \%(\mathrm{I})$ & $7,2 \%(3)$ & $\mathrm{O}, \mathrm{O} \%(\mathrm{I})$ \\
\hline $\begin{array}{l}\text { Arc-en- } \\
\text { ciel }\end{array}$ & $\mathrm{O}$ & I I & $\mathrm{o}$ & $\mathrm{O}$ & I I & $0,0 \%(2)$ & $3,6 \%(2)$ & $8,0 \%(2)$ \\
\hline Eléphant & 2 & I5 & o & $\mathrm{O}$ & I 7 & $0,0 \%(3)$ & $29,3 \%(6)$ & $37,6 \%(8)$ \\
\hline Escalier & $\mathrm{O}$ & 6 & o & o & 6 & $0,0 \%(4)$ & $59,0 \%(8)$ & $\mathrm{I} 2, \mathrm{I} \%(3)$ \\
\hline Ours & $\mathrm{O}$ & 20 & $\mathrm{o}$ & $\mathrm{O}$ & 20 & $0,0 \%(5)$ & $30,7 \%(7)$ & $2 \mathrm{I}, 6 \%(5)$ \\
\hline Ane & $\mathrm{O}$ & 27 & 43 & I & $7 \mathrm{I}$ & $3,6 \%(6)$ & I $3,7 \%(4)$ & $23,4 \%(6)$ \\
\hline Avion & 2 & I2 & O & I & I 5 & $7,7 \%(7)$ & $19,6 \%(5)$ & $38,4 \%(9)$ \\
\hline Orage & 5 & I I & o & 2 & I 8 & I $5,4 \%(8)$ & $2,2 \%(\mathrm{I})$ & $20,0 \%(4)$ \\
\hline Arbre & I & IO & $\mathrm{o}$ & 7 & I 8 & $4 \mathrm{I}, 2 \%(9)$ & $60,4 \%(9)$ & $58,2 \%(\mathrm{I} 3)$ \\
\hline Habit & $\mathrm{O}$ & 9 & $\mathrm{O}$ & IO & I9 & $52,6 \%$ (I0) & $89,7 \%(\mathrm{I} 3)$ & $34,8 \%(7)$ \\
\hline Enfant & $\mathrm{o}$ & I I & I 3 & I 4 & 38 & $56,0 \%$ (I I) & $80, \mathrm{I} \%$ (I I) & $39,6 \%$ (Io) \\
\hline Oiseau & $\mathrm{o}$ & 23 & $\mathrm{O}$ & 32 & 55 & $58,2 \%($ I 2$)$ & $66,7 \%$ (Iо) & $50,5 \%(\mathrm{I} 2)$ \\
\hline Ami & $\mathrm{O}$ & I & $\mathrm{O}$ & I I & $\mathrm{I} 2$ & $9 \mathrm{I}, 7 \%(\mathrm{I} 3)$ & $83,8 \%$ (I 2 ) & $40,6 \%$ (I I) \\
\hline
\end{tabular}

rapport est nul, valeur qui manifeste la tendance de ces 5 mots à ne provoquer que des erreurs en $/ \mathrm{n} /$.

Les deux dernières colonnes du tableau 5 contiennent deux indices distincts, qui estiment la tendance de ces I 3 mots à apparaître au pluriel plutôt qu'au singulier dans le discours environnant.

Le premier indice (colonne 8 ) est le résultat d'un test d'intuition proposé à 70 étudiants de première année de Sciences du Langage. Aucun ne connaissait ce travail sur l'acquisition des liaisons. Le matériel du test était composé de 30 noms masculins ou féminins. Vingt-cinq étaient à initiale vocalique, dont les I 3 noms figurant dans la colonne I du tableau 5 , et cinq étaient à initiale consonantique. Les sujets écoutaient chacun de ces noms dans deux syntagmes présentés successivement, l'un au pluriel et l'autre au singulier. Le syntagme au singulier était composé du nom précédé de l'article défini le, la, l' ou de l'article indéfini un ou une. Le syntagme au pluriel était formé des noms précédés du déterminant défini les ou de l'indéfini des. Au total, les sujets entendaient donc 60 paires de syntagmes nominaux, dont 30 de type indéfini - un ami-des amis - et 30 de type défini - l'ami-les amis. Ces 60 paires étaient lues par l'enquêteur dans un ordre aléatoire. Pour I 5 mots, l'ordre singulierpluriel était utilisé dans les paires de type défini et l'ordre pluriel-singulier dans les paires de type indéfini. Pour les Is autres mots, la correspondance entre le type défıni-indéfini et l'ordre de présentation du singulier et du pluriel étaient inversés.

Pour chaque paire de type un ami-des amis ou de type l'ami-les amis, chacun des sujets devait juger lequel parmi les deux syntagmes - singulier ou pluriel - il estimait entendre le plus souvent. Chaque nom donnait donc lieu à I 40 jugements, puisqu'il était jugé par 70 sujets dans une paire de type indéfini et une paire de type défini. La colonne 8 du tableau 5 donne le rapport du nombre de jugements 
«pluriel» au nombre total de jugements effectifs. Par exemple, le mot ami a été proposé I 40 fois au jugement des sujets, I I 4 jugements ont privilégié le pluriel et

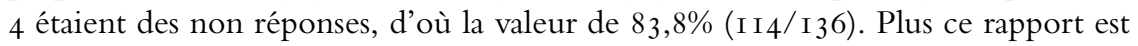
élevé et plus les sujets ont l'intuition qu'un nom est utilisé au pluriel.

Afin de vérifier la validité de ces jugements, nous avons calculé un second indice (colonne 9) basé sur l'observation de l'orientation des 30 noms du test vers le pluriel ou vers le singulier dans la base textuelle Frantext. Cette base est constituée d'environ 3500 textes, ouvrages littéraires et scientifiques, parus lors des cinq derniers siècles. Pour chacun des noms du test, nous avons cherché la fréquence des syntagmes nominaux définis et indéfinis soumis au jugement des auditeurs. La colonne 9 du tableau 5 liste pour chaque mot le rapport entre le nombre d'occurrences des syntagmes au pluriel d'une part, et le nombre d'occurrences des syntagmes au singulier additionné au nombre d'occurrences des syntagmes au pluriel d'autre part. Ainsi, dans Frantext, le mot ami apparait 5753 fois au singulier (3499 fois avec l'indéfini singulier $u n$ et 2254 fois avec le défini le) et 3925 fois au pluriel (2422 fois avec des et $\mathrm{I} 5 \mathrm{O} 3$ fois avec les). Le rapport de la colonne 9 vaut donc $40,6 \%(3925 / 3925+5753)$. La moyenne de ce rapport pour les 30 noms du test est de $35 \%$ et pour 23 de ces noms, sa valeur est inférieure à 50\%. Cette distribution signifie que les noms sont majoritairement intégrés à des syntagmes au singulier, ce qui pourrait expliquer que les erreurs de liaison en /n/ sont globalement plus fréquentes (Dugua, 2002). Toutefois, certains noms sont moins orientés que d'autres vers le singulier (par exemple, arbre, oiseau et ami). Relativement aux autres, ils sont donc plus souvent précédés des déterminants les ou des, et donc de la liaison $/ z /$. Cette orientation relative vers le pluriel dépendant des propriétés sémanticoréférentielles des noms, il est improbable qu'elle differe radicalement dans le corpus d'écrits Frantext et dans l'environnement langagier oral.

Dans le tableau 5, les I 3 noms sont rangés dans l'ordre croissant des valeurs du rapport indiquant leur tendance à provoquer les erreurs en /z/ (colonne 7). Les ordres à l'intérieur de chacune des colonnes 7, 8 et 9 sont indiqués entre parenthèses. Ces ordres ne sont pas identiques mais suffisamment proches pour que les corrélations de Spearman soient significatives malgré le faible nombre d'items impliqués. La corrélation entre la tendance de l'enfant S. à produire des erreurs en $/ \mathrm{z} /$ (colonne 7) et la tendance des adultes à juger les mots orientés vers le pluriel dans le test d'intuition (colonne 8) vaut $0.656(\mathrm{p}=0.023 \mathrm{I})$. La corrélation entre la tendance de S. à produire des erreurs en $/ \mathrm{z} /$ (colonne 7) et l'orientation vers le pluriel des noms dans Frantext (colonne 9) vaut $0.763(\mathrm{p}=0.0082)$. Enfin, la corrélation entre l'orientation vers le pluriel dans le test d'intuition (colonne 8) et dans Frantext (colonne 9) vaut 0.659 ( $\mathrm{p}=0.0224$ ). Plus les noms sont orientés vers le pluriel dans l'intuition des locuteurs, plus ils sont précédés des déterminants les et des inducteurs de la liaison /z/ dans le corpus Frantext et plus ils sont précédés d'erreurs de liaison en /z/ chez l'enfant S.

Cette relation entre la nature phonétique des liaisons enfantines et le nom qui les suit est encore plus apparente dans les mots du corpus de S. dont la forme au singulier est différente de la forme au pluriel. Trois de ces mots sont présents 
Tableau 6. Erreurs entre 2 et 6 ans chez S. et formes du pluriel ou du singulier des mots cuf et oil

\begin{tabular}{lcccc}
\hline \hline & $\begin{array}{l}\text { Erreurs en } \\
/ \mathrm{n} /\end{array}$ & $\begin{array}{l}\text { Erreurs en } \\
/ \mathrm{z} /\end{array}$ & $\begin{array}{l}\text { Erreurs en } \\
/ \mathrm{l} /\end{array}$ & $\begin{array}{l}\text { Erreurs en } \\
/ \mathrm{t} /\end{array}$ \\
\hline Forme du singulier: [œe], [œj] & $5 \mathrm{I}$ & 5 & 2 & 0 \\
Forme du pluriel: $[\phi],[\mathrm{j} \phi]$ & 4 & 28 & 0 & 0 \\
\hline \hline
\end{tabular}

dans le recueil: $\propto u f$ (40 occurrences), eil (44 occurrences), animal (4 occurrences). Dans le tableau 6, sont représentées les possibilités de combinaisons entre les erreurs enfantines et les formes des mots ouf et oil, dont l'effectif est au moins égal à 6 . Chacune des consonnes impliquées dans les erreurs $-/ \mathrm{n} / \mathrm{,} / \mathrm{z} / \mathrm{l} / \mathrm{t} /$ ou $/ \mathrm{l} / \mathrm{-peut}$ en effet être combinée avec les formes du singulier ([œf], [œj]) ou avec les formes du pluriel $([\phi],[j \phi])$.

Le tableau 6 fait apparaitre un lien significatif $\left(\mathrm{Chi}^{2}=62, \mathrm{DDL}=2, \mathrm{p}<0.000 \mathrm{I}\right)$ entre les erreurs et les formes de mots. On constate plus précisément que les formes du singulier des mots cuf et ceil sont associées à l'erreur en /n/, alors que les formes du pluriel sont associées à l'erreur en /z/. En d'autres termes, les séquences $[\mathbf{z} \phi],[\mathrm{zj} \phi]$, [nœf], [nœj] sont fréquentes puisqu'elles représentent 79 cas d'erreurs sur 90, alors que les séquences [n $\phi]$, [njø], [zœf], [zœj] sont rares. Dans l'environnement langagier, les formes de mot du pluriel sont plus souvent précédées de déterminants au pluriel qui induisent une liaison /z/ et les formes de mots au singulier de déterminants au singulier qui induisent la liaison /n/. Dans les erreurs qu'elle commet, S. reproduit donc les séquences liaison-mot2 qu'elle a entendues.

Les résultats sur l'orientation en nombre des noms et sur les items lexicaux dont la forme varie avec le nombre sont convergents. La nature phonétique /z/ ou /n/ des erreurs qui précèdent une forme dépend des déterminants les plus probables devant cette forme. Plus un nom ou une forme de nom est précédé de déterminants inducteurs de la liaison / $\mathrm{z} /$ dans l'environnement langagier et plus les erreurs en /z/ sont fréquentes devant cette unité dans le corpus de S. L'enfant établit donc une relation entre la nature phonétique des liaisons et la forme phonologique du mot qui les suit dans les énoncés qu'elle rencontre.

A nouveau, ces données acquises en situation naturelle confirment totalement des résultats obtenus auprès d'un très grand nombre de sujets lors de tâches expérimentales. Dugua (en préparation) a rassemblé les données de plusieurs expériences de dénomination provoquant la production de noms après les déterminants un et deux chez des enfants âgés de 2 à 6 ans. Au total, elle dispose de données sur 9 noms masculins, chacun de ces noms ayant été produit par un nombre d'enfants qui varie entre I23 et $5 \mathrm{I} 2$. En utilisant le même rapport que celui que nous avons calculé dans le tableau 5 (rapport $z / z+n$, colonne 7 ), elle a estimé la tendance de ces noms à être précédés d'erreurs en /z/ dans les productions enfantines. La corrélation entre ce rapport et la propension de ces noms à apparaître au pluriel dans le corpus Frantext ne montre qu'une tendance (Spearman $=0.633$, 
$\mathrm{p}=0.0732$ ). En revanche, ce rapport corrèle significativement avec les jugements d'intuition sur l'orientation en nombre des noms (Spearman $=0.783, \mathrm{p}=0.0267$ ).

\section{DISCUSSION ET CONCLUSION}

Les trois études empiriques visaient deux objectifs. Premièrement, il s'agissait d'observer l'usage enfantin des liaisons dans des situations naturelles pour valider et préciser les repères développementaux établis antérieurement par des tâches expérimentales. Deuxièmement, nous voulions utiliser cette extension des données pour départager deux conceptions concurrentes de l'acquisition des liaisons: une conception exemplariste basée sur l'usage et une conception fondée sur la connaissance de principes universels. La discussion qui suit examinera successivement ces deux objectifs.

\subsection{L’apport de données recueillies en situation naturelle}

Les cinq études de cas transversales montrent que le taux de réalisation des liaisons obligatoires atteint entre 4 et 5 ans la cible adulte de I00\%. Le suivi longitudinal de deux enfants confirme ce résultat mais fait apparaitre une variabilité interindividuelle: seul l'enfant dont les parents ont fait des études supérieures atteint cette valeur maximale entre 40 et 50 mois. Selon notre interprétation, les enfants de milieu favorisé entendent davantage de parole adulte (Hoff, 2002) et ils rencontrent donc plus fréquemment des séquences moti-mot 2 incluant une liaison bien formée. Un deuxième effet de fréquence sur les liaisons obligatoires est mis en évidence du fait de la diversité des contextes recueillis dans les corpus transversaux. La liaison après un pronom clitique progresse plus vite que celle qui suit un déterminant, le premier contexte étant plus courant que le second dans les énoncés des enfants et des parents enregistrés.

Les liaisons facultatives sont réalisées dans 5\% des cas par l'enfant de 3-4 ans participant à l'étude transversale et dans I $2 \%$ des cas chez celui de I 2 ans, valeur proche de la cible adulte en situation familière (Iо\%). L'étude longitudinale montre que leur réalisation augmente entre 40 et 50 mois, mais uniquement chez l'enfant dont les parents ont fait des études supérieures. Comme c'est le cas pour les liaisons obligatoires, l'acquisition des facultatives est le lieu de variations interindividuelles liées à l'origine sociale.

Les résultats acquis par l'étude transversale et le suivi longitudinal confirment en général les données expérimentales résumées dans Chevrot, Dugua et Fayol (2005). Un résultat notable établi par les deux méthodologies est l'incidence particulière des différences sociologiques dans le développement des deux types de liaison. Dans le cas des liaisons obligatoires, les écarts entre enfants de milieux différents s'estompent progressivement lorsque l'âge augmente. En effet, les parents de tous les milieux réalisent toutes les liaisons obligatoires. Comme le prédit le modèle basé sur l'usage, tous les enfants disposeront donc, après un temps plus ou moins long, d'une connaissance des relations standard motI-mot2 suffisante pour 
généraliser un schéma de type des $+/ \mathrm{zX} /$ ou $u n+/ \mathrm{nX} /$. C'est la quantité totale de liaisons correctes entendues, liée à la quantité de parole adressée à l'enfant, qui déterminerait la durée nécessaire pour former le stock lexical permettant cette généralisation.

Dans le cas des liaisons facultatives, la réalisation des adultes est variable et sociodifférentielle et l'enfant entend chaque séquence moti - mot2 avec ou sans liaison. La probabilité qu'il entende plus souvent l'une ou l'autre des variantes dépend du milieu dans lequel il évolue et détermine à terme leur disponibilité respective. Plus un enfant grandit, plus il a été exposé longtemps aux fréquences de réalisation typiques de son milieu, et plus son usage traduit l'apprentissage culturel de normes d'usage. Il est donc attendu que les différences sociologiques s'accroissent avec l'âge dans le cas d'un marqueur sociolinguistique tel que les liaisons optionnelles.

\subsection{La confrontation des modèles}

La diversité des données recueillies dans des situations d'interaction a permis de confronter sur deux points les modèles présentés en introduction. Dans les deux cas, les faits confirment les prédictions du modèle exemplariste basé sur l'usage. Nous allons rappeler ces faits puis examiner dans quelle mesure ils sont compatibles avec le modèle concurrent.

Le premier résultat concerne l'acquisition précoce des liaisons obligatoires chez l'enfant de 3 ans observé dans l'étude transversale. Plus cet enfant produit fréquemment une séquence particulière motI-mot2 où le motI est un clitique ou un déterminant, plus il réalise de liaisons correctes à l'intérieur de cette séquence. Ce résultat découle directement du modèle basé sur l'usage, dont la deuxième étape consiste en l'apprentissage des relations entre certains mots I et les variantes adéquates du mot2 (un + /nurs/, des + /zurs/, etc.). Dans le modèle de lexique que propose Bybee (I995), les séquences de mots fréquentes sont mémorisées. L'enfant mémoriserait donc des suites fréquentes incluant un clitique ou un déterminant suivis de la variante adéquate du mot2. Lors de la production, ces séquences sont activées globalement, avec la liaison correcte qu'elles contiennent. Puisque les erreurs découlent de la combinaison d'un moti avec une variante inadéquate du mot2, l'activation de séquences globales évite cette combinatoire et supprime les raisons mêmes de l'erreur.

Dans le modèle basé sur la connaissance de principes universaux, la fréquence des séquences moti-mot2 ne joue aucun rôle dans la maîtrise des liaisons. Dans ce modèle, c'est la proximité entre certaines consonnes de liaison et certaines consonnes morphologiques qui conduirait le jeune locuteur à rattacher la liaison sous forme de segment flottant à la finale du moti et ce rattachement éliminerait les erreurs. Toutefois, nos données ne sont pas compatibles avec cette proposition. Parmi les clitiques et les déterminants fréquents recueillis dans le corpus de l'enfant de 3 ans (tableau 3), seul l'article un est relié à des formes fléchies ou dérivées qui contiennent une consonne /n/ identique à la liaison (la forme du féminin une, l'adjectif unique, etc.). Malgré le statut morphologique particulier de un, le record 
de liaisons justes à 3 ans ne lui revient pas. Ce record revient en fait au clitique en, qui est caractérisé par son insertion dans des séquences motI-mot2 récurrentes mais qui n'est rattaché à aucune forme fléchie ou dérivée. Nos résultats sont donc incompatibles avec l'idée d'une intervention précoce de la morphologie dans la capacité à produire des liaisons obligatoires justes.

Le second résultat permettant la confrontation entre les deux modèles provient de l'étude des erreurs de liaison recueillies chez l'enfant S. entre 2 et 6 ans. Les mots qui sont jugés typiques d'un usage au pluriel par des adultes et qui sont précédés plus souvent des déterminants les et des dans le corpus Frantext donnent lieu à davantage d'erreurs consistant à remplacer la liaison attendue /n/ par un /z/. De la même façon, les formes au pluriel $[\phi]$, [j $\phi]$ des mots $\propto u f$ et $\propto i l$ sont généralement précédées d'erreurs impliquant la consonne /z/, alors que les formes au singulier [œf] et [œj] des mêmes mots déclenchent des erreurs en $/ \mathrm{n} /$. Ce double résultat découle de l'étape I du modèle basé sur l'usage. Lors de cette étape, l'enfant récupère plusieurs exemplaires lexicaux de chacun des mots2 en répétant une segmentation favorisant $\mathrm{CV}$ initial dans les différents contextes de liaison où ces mots apparaissent. La liste des exemplaires récupérés pour un mot donné (/nami/, /zami/, /tami/ ou /ami/ pour le mot ami) dépend des contextes où ce mot a été rencontré et elle n'est donc pas nécessairement complète. Ainsi, un mot souvent utilisé au pluriel (ami) ou une forme de mot spécifique de ce nombre (la forme $[\phi]$ du mot $\alpha u f$ ) sont entendus dans des séquences où ils sont précédés d'un déterminant au pluriel. L'enfant les rencontre donc souvent après une liaison /z/ et il est probable qu'il récupère l'exemplaire de ces mots commençant par /z/. L'utilisation ultérieure de cet exemplaire en $/ \mathrm{z} /$ avec le déterminant $u n$ aboutira à une erreur de remplacement de $/ \mathrm{n} / \mathrm{par} / \mathrm{z} /$.

En outre, même si l'enfant mémorise l'exemplaire en $/ z /$ et l'exemplaire en /n/ de mots ou de formes de mots spécifiques du pluriel (/zjф/ et /nœj/, /zami/ et /nami/), il percevra plus fréquemment l'exemplaire en $/ z /$ de ces mots dans l'environnement langagier. Cet exemplaire sera donc renforcé et deviendra plus disponible. De ce fait, il émergera plus facilement, notamment dans les contextes où sa présence aboutit à une erreur en $/ \mathrm{z} /$.

Le modèle basé sur la connaissance de principes universaux accorde aussi une place aux effets de fréquence. Dans la seconde étape, l'enfant segmente les gabarits de l'étape précédente en se basant sur le Principe de Maximisation des Attaques, qui le conduit à encoder une position consonantique abstraite - sans contenu phonétique - à l'initiale du nom. L'enfant assigne ensuite un contenu phonétique à cette position, par différents moyens, dont le remplissage par la consonne /n/, la plus fréquente des liaisons obligatoires: « (l'enfant) essaie de remplir la position prosodique d'attaque avec toutes les consonnes qu'il a rencontrées dans ce contexte. Ceci expliquerait le pourcentage plus élevé de "n" » (Wauquier-Gravelines et Braud, 2005: 62). Même s'il est indéniable que la nasale est la consonne la plus intrusive dans les erreurs (Dugua, 2002), les données sur les noms et formes de mots orientés vers le pluriel montrent que la nature phonétique des liaisons erronées est sensible au mot2: la fréquence du /n/ diminue et celle du / $/$ / augmente dans le 
cas des noms souvent précédés de la liaison /z/. Ainsi, l'incidence relative du /n/ et $\mathrm{du} / \mathrm{z} /$ dépend du lien statistique qui unit une consonne de liaison particulière à un mot2 particulier. Ce résultat ne semble pas pouvoir être intégré au modèle basé sur la connaissance de principes universaux sans en altérer les fondements. Si on admet que la position consonantique abstraite située à l'initiale d'un nom particulier est liée au contenu phonétique de la consonne de liaison qui précède le plus souvent ce nom particulier, alors cette consonne initiale et son contenu phonétique sont spécifiques d'un item lexical particulier et ils n'ont plus rien d'abstrait.

Les données recueillies dans des situations naturelles d'interaction confortent le modèle basé sur l'usage. Parmi les forces qui guident l'encodage lexical des liaisons dans des sens contradictoires, la syllabisation avec le mot suivant joue précocement un rôle central. Les enfants positionnent une frontière de mot devant l'attaque des syllabes CV. La nature exemplariste du lexique qu'ils construisent leur permet par ailleurs de prendre en compte la nature alternante de la consonne de liaison en mémorisant plusieurs variantes des mots2. Ces variantes sont concrètes au sens où chacune d'elle est dotée d'un contenu phonétique spécifique. Les jeunes locuteurs apprennent ensuite les contingences statistiques entre le mot précédent et les variantes adéquates. Et ce mode d'apprentissage les conduit à produire d'abord les liaisons correctes dans des séquences fréquentes.

La portée des résultats obtenus dans cet article s'arrête à ce stade. D'autres données suggèrent que les jeunes locuteurs élaborent ultérieurement des constructions qui généralisent la relation entre certains mots et une classe de variantes caractérisée par une consonne de liaison particulière (voir section 2.I). A ce niveau, les contraintes morphologiques et le rôle de la graphie pourraient entrer en scène et modifier l'impact initial de la syllabisation. Quelle que soit l'issue de ce stade plus tardif, un fait est certain et devra être pris en compte dans le débat sur le statut lexical des liaisons (Côté, 2005): le point de départ à partir duquel démarre le chemin qui mène à la compétence adulte est le rattachement précoce des liaisons à l'initiale d'exemplaires en alternance dans le lexique enfantin.

Adresse pour correspondance:

Jean-Pierre Chevrot

Université Grenoble 3

BP 25, 38040 Grenoble Cedex

France

e-mail:jpchevrot@wanadoo.fr

\section{REFERENCES}

Agren, J. (1973). Etude sur quelques liaisons facultatives dans le français de conversation radiophonique: fréquences et facteurs. Uppsala, Stockholm: Almqvist och Wiksell.

Ahmad, M. (I993). Vingt heures de français parlé. Aspects phonétiques de la liaison. Thèse de doctorat, Université Grenoble 3, Grenoble. 
Alexander, J. (2004). Frequency, prosody, and French liaison: Testing Bybee's hypothesis. BA Dissertation, Boston University.

Basset, B. (2000). La liaison à 3, 7 et I I ans: description et acquisition. Mémoire de maitrise, Universite Grenoble 3.

Boë, L.-J. et Tubach, J.-P. (I992). De A à Zut, dictionnaire phonétique du français parlé. Grenoble: Ellug.

Booij, G. et de Jong, D. (1987). The domain of liaison: theories and data. Linguistics, 25: $1005-1025$.

Bybee, J. (1995). Regular morphology and the lexicon. Language and Cognitive Processes, IO: $425-455$.

Bybee, J. (200I). Frequency effects on French Liaison. Dans: J. Bybee and P. Hopper (dir.), Frequency and the Emergence of Linguistic Structure, Amsterdam: John Benjamins, pp. 337-359.

Bybee, J. (2005). La liaison: effets de fréquence et constructions. Langages, I 58: 24-37.

Chabanal, D. (2003). Un aspect de l'acquisition du français oral: la variation sociophonétique chez l'enfant francophone. Thèse de doctorat, Université Montpellier 3.

Chevrot, J.-P. et Fayol, M. (200I). Acquisition of French liaison and related child errors. Dans: M. Almgren, A. Barreña, M. J. Ezeizabarrena, I. Idiazabal, et B. MacWhinney (dir.), Research on Child Language Acquisition, volume 2. Somerville, MA: Cascadilla Press, pp. 760-774.

Chevrot, J.-P., Dugua, C. et Fayol, M. (2005). Liaison et formation des mots en français: un scénario développemental. Langages, I 58: 38-52.

Content, A., Kearns, R. K. et Frauenfelder, U. H. (200I). Boundaries versus onsets in syllabic segmentation. Journal of Memory and Language, 45: I77-I99.

Côté, M.-H. (2005). Le statut lexical des consonnes de liaison. Langages, I 58: 66-78.

De Jong, D. (I994). La sociophonologie de la liaison orléanaise. Dans: C. Lyche (dir.), French Generative Phonology: Retrospective and Perspectives. London: AFLS/ESRI, pp. 95-I30.

Dugua, C. (2002). Liaison et segmentation du lexique en français: vers un scénario développemental. Diplôme d'Etudes Approfondies, Université Grenoble 3.

Dugua, C. (2005). De la liaison à la formation du lexique chez les jeunes enfants francophones. Le Langage et l'Homme, 40.2: I63-I82.

Dugua, C. (en préparation). Les étapes de l'acquisition de la liaison et la formation du lexique chez les enfants francophones entre 2 et 6 ans. Thèse de doctorat, Université Grenoble 3.

Encrevé, P. (1988). La liaison avec et sans enchaînement. Phonologie tridimensionnelle et usages du français. Paris: Seuil.

Gaatone, D. (I979). Liaison et structure syllabique du français. Le Français Moderne, 4: 3 I $2-334$.

Grégoire, A. (1937). L'apprentissage du langage, vol. 1: les deux premières années. Paris: Droz.

Hoff, E. (2002). Causes and consequences of SES-related differences in parent-to-child speech. Dans: M. H. Bornstein and R. H. Bradley (dir.), Socioeconomic Status, Parenting, and Child Development. Mahwah, NJ: Lawrence Erlbaum Associates, pp. I47I60.

Kemmer, S., Barlow, M. et (2000). Introduction: A usage-based conception of language. Dans: M. Barlow and S. Kemmer (dir.), Usage-based Models of Language Use. Stanford, CA: CSLI Publications, pp. vii-xxvii. 
Klausenburger, J. (I974). Rules Inversion, opacity, conspiracies: French liaison and elision. Lingua, 34.2-4: 167-173.

Laks, B. (2005). La liaison et l'illusion. Langages, I 58: I0 I-I25.

Lieven, E., Behrens, H., Speares, J. et Tomasello, M. (2003). Early syntactic creativity: a usage-based approach. Journal of Child Language, 30: 333-370.

Lucci, V. (1983). Etude phonétique du français contemporain à travers la variation situationnelle. Grenoble: Publications de l'Université Grenoble 3.

Malécot, A. (1975). French liaison as a function of grammatical, phonetic and paralinguistic variables. Phonetica, 32: I6I-I79.

Mattys, S. L. et Jusczyk, P. W. (200I). Do infants segment words or recurring continuous patterns? Journal of Experimental Psychology: Human Perception and Performance, 27: 644655 .

Meradji, O. et Grégoire, G. (200I). Les liaisons phonétiques au sein d'une famille. Mémoire de maitrise. Université Grenoble 3.

Moisset, C. (2000). Variable liaison in Parisian French. PhD thesis, University of Pennsylvania.

Morin, Y. C. (2003) [1998]. Remarks on prenominal liaison consonants in French. Dans: S. Ploch (dir.), Living on the Edge, 28 Papers in Honour of Jonathan Kaye. Amsterdam: Mouton de Gruyter, pp. 385-400.

Morin, Y. C. (2005). La liaison relève-t-elle d'une tendance à éviter les hiatus? Réflexions sur son évolution historique. Langages, i 58: 8-23.

Nardy, A. (2003). Production et jugement d'acceptabilité entre 2 et 6 ans: aspects psycholinguistiques et sociolinguistiques de l'acquisition des liaisons. Diplôme d'Etudes Approfondies, Université Grenoble 3.

Tomasello, M. (2000). The item-based nature of early syntactic development. Trends in Cognitive Science, 4.4: I 56-I63.

Tomasello, M. (2003). Constructing a Language: A Usage-based Theory of Language Acquisition. Cambridge, MA and London: Harvard University Press.

Tranel, B. (2000). Aspects de la phonologie du français et la théorie de l'optimalité. Langue française, I26: 39-72.

Vihman, M. (1996). Phonological Development: The Origins of Language in the Child. Oxford: Blackwell.

Wauquier-Gravelines, S. et Braud, V. (2005). Proto-déterminant et acquisition de la liaison obligatoire en français. Langages, I 58: 53-65.

Wauquier-Gravelines, S. (2005). Statut des représentations phonologiques en acquisition, traitement de la parole continue et dysphasie développementale. Habilitation à Diriger des Recherches, Ecole des Hautes Etudes en Sciences Sociales, Paris. 